\title{
Uniportal video-assisted thoracic surgery reduced the occurrence of post-thoracotomy pain syndrome after lobectomy for lung cancer
}

\author{
Kyoji Hirai ${ }^{1}$, Jitsuo Usuda ${ }^{2}$ \\ ${ }^{1}$ Department of Thoracic Surgery, Nippon Medical School Chiba Hokusoh Hospital, Inzai, Chiba, Japan; ${ }^{2}$ Department of Thoracic Surgery, Nippon \\ Medical School, Tokyo, Japan \\ Contributions: (I) Conception and design: K Hirai; (II) Administrative support: None; (III) Provision of study materials or patients: K Hirai; (IV) \\ Collection and assembly of data: K Hirai; (V) Data analysis and interpretation: All authors; (VI) Manuscript writing: All authors; (VII) Final approval \\ of manuscript: All authors. \\ Correspondence to: Kyoji Hirai, MD. Division of thoracic surgery, Nippon Medical School Chiba Hokusoh Hospital, 1715 Kamakari, Inzai, Chiba \\ 270-1674, Japan. Email: ky-hirai@nms.ac.jp.
}

\begin{abstract}
Background: Post-thoracotomy pain syndrome (PTPS) is difficult for thoracic surgeons to manage. PTPS should never arise after minimally invasive surgery (MIS). Uniportal video-assisted thoracoscopic surgery (U-VATS), a form of MIS for thoracic disease, has become more common around the world and might reduce the risk of PTPS after thoracic surgery. We compared the frequencies of PTPS after U-VATS-based and multi-port VATS (M-VATS)-based lobectomy for lung cancer.

Methods: We compared the data for 142 and 70 cases in which U-VATS- and M-VATS-based lobectomies were performed, respectively. General surgical outcomes, the numeric rating scale (NRS) score, analgesic usage, and neuropathic pain according to the pain DETECT questionnaire (PDQ) were assessed. The presence of $>1$ of the following items at two postoperative months (POM) was used to diagnose PTPS: (I) an NRS score of $>3$, (II) analgesic use, and (III) exhibiting $>1$ of a list of 7 symptoms according to the PDQ.

Results: There were no significant intergroup differences in the operation time, intraoperative blood loss, the number of dissected lymph nodes, or the duration of the drainage period or hospital stay. In the U-VATS and M-VATS groups, the mean NRS score during two postoperative months was $0.5 \pm 0.1$ and $1.5 \pm 0.3$, respectively $(\mathrm{P}<0.01)$, and analgesics were used for a mean of $7.3 \pm 1.5$ and $13.3 \pm 1.8$ days, respectively, during the postoperative 2 months $(\mathrm{P}<0.01)$. As for the frequency of PTPS-related symptoms on postoperative month (POM) 2, allodynia (U-VATS: $2.1 \%$ vs. M-VATS: $10 \%, \mathrm{P}=0.028)$, hypoesthesia $(2.1 \%$ vs. $10 \%$, $\mathrm{P}=0.028)$, and numbness (1.4\% vs. $8.6 \%, \mathrm{P}=0.029)$ often arose in both groups. PTPS occurred in $2.8 \%$ and $11.4 \%$ of the patients in the U-VATS and M-VATS groups, respectively $(\mathrm{P}=0.025)$.
\end{abstract}

Conclusions: Compared with M-VATS, U-VATS exhibited a significantly lower incidence of PTPS.

Keywords: Uniportal video-assisted thoracic surgery (U-VATS); multiport VATS (M-VATS); post-thoracotomy pain syndrome (PTPS); lobectomy; early lung cancer

Submitted May 05, 2019. Accepted for publication Aug 06, 2019.

doi: $10.21037 /$ jtd.2019.09.07

View this article at: http://dx.doi.org/10.21037/jtd.2019.09.07

\section{Introduction}

Post-thoracotomy pain syndrome (PTPS) is a difficult complication for thoracic surgeons to control. In PTPS, pain and anxiety continue after discharge, and many patients complain of symptoms. Anti-inflammatory analgesics are ineffective in many cases, and outpatient consultations are required to explain the condition to patients. The developmental mechanism underlying PTPS is considered 
to involve intercostal neuropathy; i.e., neuropathic pain, but mental and social factors are also considered to contribute to the condition (1).

Uniportal video-assisted thoracoscopic surgery (U-VATS) is a less invasive surgical procedure, and it is used to treat respiratory surgical diseases in some countries. The associated healthcare costs are low compared with those associated with robot-assisted thoracoscopic surgery (RATS), and U-VATS can be performed at many medical facilities, as it only requires the instruments used for conventional multi-port VATS (M-VATS) plus specific forceps. However, the U-VATS procedure is slightly more complex for operators than the M-VATS procedure. Since a scope, forceps, and scissors are simultaneously inserted through a $3-4-\mathrm{cm}$ thoracotomy wound, U-VATS requires the use of devices that prevent interference among the instruments. Although U-VATS is technically difficult compared with conventional M-VATS, many studies have reported that similar surgical outcomes can be achieved by learning the procedure (2-4). U-VATS has been reported to reduce wound pain early after surgery $(2,5,6)$, but no previous studies have investigated its effects on the risk of PTPS, compared with that seen after conventional M-VATS. We investigated this issue in the present study.

\section{Methods}

The study was approved by the institutional review board and the committee of Nippon Medical School Chiba Hokusoh Hospital. A retrospective review of a prospectively maintained database identified 212 patients with clinical stage I non-small cell lung cancer who underwent curative thoracoscopic surgery at Nippon Medical Chiba Hokusoh Hospital between June 2012 and March 2019. U-VATS and M-VATS were performed in 142 and 70 patients, respectively, by the same thoracic surgeon. In our study, considering the learning curve of U-VATS, I compared U-VATS and M-VATS after performing initial 20 lobectomies of U-VATS. Informed consent to use their data was obtained from all patients before surgery.

\section{Anaesthesia and analgesia}

All patients received a combination of epidural and general anaesthesia, and were provided with

patient-controlled analgesia after operation. The postoperative analgesia is performed with intravenous morphine administered through Patient Controlled-
Analgesia delivery. Morphine $1 \mathrm{mg}$ was given for each request and continuous infusion was at a rate of $1 \mathrm{mg} / \mathrm{h}$. Patient had a 10 min lockout period and a safe higher limit of $20 \mathrm{mg}$ in 4 hours. After the first 24 hours, epidural catheter was removed and the administration of intravenous morphine was stopped. According to the patient's symptoms, analgesics such as NSAIDS, pregabalin and tramadol hydrochloride was adequately used.

\section{Surgical procedures}

In the U-VATS procedure, an $\sim 5$-cm incision was made along the anterior (upper and lower lobectomy) or middle (lower lobectomy) axillary line, before a Wrap Protector mini was attached (Hakko Inc., Japan). Procedures for protecting the intercostal nerves were conducted by the surgeon and the assistants. The U-VATS procedure was performed as atraumatically as possible; i.e., attempts were made to prevent contact between the thoracoscope or forceps and the intercostal nerves. The thoracoscope was mostly handled at an angle of $>45$ degrees from the chest wall. The surgery was carried out using a $10-\mathrm{mm}$ or 5-mm, 30-degree, oblique-viewing thoracoscope and facing/inverted dual monitors without any specific devices. The Wrap Protector mini was used to open incisions, and no small rib retractors were used. Trocars (Endopath, 12-mm, Ethicon, USA; Thoracoport, $5.5 \mathrm{~mm}$, Medtronic, USA) were used in the ports in the M-VATS procedure. An energy device, a HARMONIC scalpel (Ethicon, USA), was used for mediastinal lymph node dissection. Various surgical factors (surgical outcomes), the incidence of complications, postoperative complications (prolonged air leakage, pneumonia, atelectasis, and wound infections), and 60-day mortality were examined. Postoperative wound pain was monitored using the numeric rating scale (NRS). The NRS score was assessed on postoperative day 7,30 , and 60 . The number of days on which analgesics were used within the first two months after the operation was also determined. Non-steroidal anti-inflammatory drugs, pregabalin, and tramadol hydrochloride were administered as analgesic agents. In addition, neuropathic pain was assessed in detail using the painDETECT questionnaire (PDQ) (7). PTPS was diagnosed based on the presence of more than one of the following items according to the PDQ on postoperative month 2 : (I) an NRS score of $>3$; (II) analgesic use; and (III) exhibiting more than one of the following 7 symptoms: allodynia, hypoesthesia, hyperalgesia, numbness, a prickling sensation, a burning sensation, and an aching sensation. 
Table 1 Clinical characteristics of study population

\begin{tabular}{lccc}
\hline Characteristics & M-VATS $(\mathrm{n}=70)$ & U-VATS $(\mathrm{n}=142)$ & P value \\
\hline Age (years) & $64 \pm 8$ & $66 \pm 8$ & 0.74 \\
Sex & & & 0.79 \\
Male & $42(60.0)$ & $92(64.8)$ & \\
Female & $28(40.0)$ & $50(35.2)$ & \\
Tumor size (cm) & $2.4 \pm 0.6$ & $2.8 \pm 0.7$ & 0.74 \\
FEV1.0 (L) & $1.7 \pm 0.4$ & $1.9 \pm 0.6$ & 0.32 \\
Histology & & & 0.46 \\
SC & $16(22.9)$ & $39(27.5)$ & \\
AD & $54(77.1)$ & $103(72.5)$ & \\
Fissure statement & & & 0.66 \\
Developed & $43(61.4)$ & $98(69.0)$ & \\
Not well developed & $27(38.6)$ & $44(31.0)$ & \\
\hline
\end{tabular}

Data are presented as mean \pm SD or number (percentage). SD, standard deviation; M-VATS, multi-port video-assisted thoracoscopic surgery; U-VATS, uniportal video-assisted thoracoscopic surgery; FEV1.0, forced expiratory volume in $1 \mathrm{~s}$; $\mathrm{SC}$, squamous cell carcinoma; $A D$, adenocarcinoma.

Allodynia was evaluated based on a detailed interview and tests with an ink brush.

\section{Statistical analysis}

Data were expressed as mean \pm standard deviation. Student's $t$ test was applied for continuous variables and Chi-square test used for categorical variables. All data were analyzed using the software SPSS version 17 for Windows (IBM Corp, Armonk, NY, USA). P values of $<0.05$ were considered to indicate significance for all parameters.

\section{Results}

\section{Preoperative data and resected lobe of lung}

From 2012 to 2018, we performed 142 lobectomies via U-VATS and 70 lobectomies via M-VATS for clinical stage I lung cancer. The patients' preoperative characteristics are summarized in Table 1. As shown in Table 1, there were no significant intergroup differences with regard to age, sex, tumor size, forced expiratory volume in $1.0 \mathrm{~s}$ (FEV 1.0), histology, or the affected pulmonary lobes.
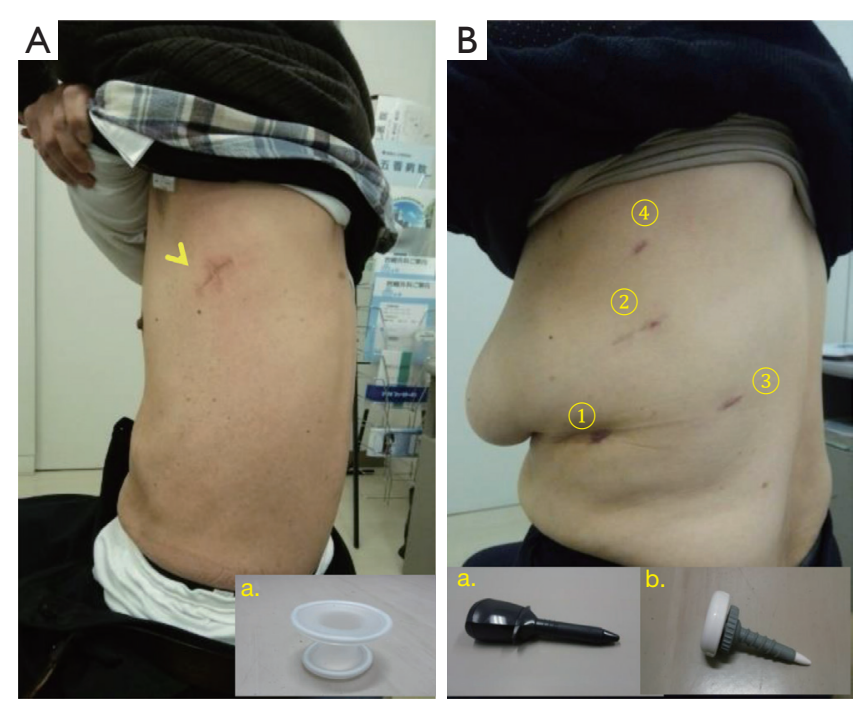

Figure 1 Skin incision 1 month after lobectomy in U-VATS and M-VATS. (A) U-VATS: upper/middle lobectomy: 4th intercostal level, anterior axillary line; lower lobectomy: 5 th intercostal level, middle axillary line. Mean wound size: $4.2(3.0-5.0) \mathrm{cm}$. a, Wrap protector mini. (B) M-VATS: (I) $1.0-\mathrm{cm}$ incision, 8 th intercostal space (ICS), mid-axillary line (12-mm trocar); (II) 3-4 cm incision, 5 th or 6th ICS, anterior axillary line (Wrap protector); (III) $1.0-\mathrm{cm}$ incision, 7th ICS along the posterior axillary line (12-mm trocar); (IV) When needed, 1.0-cm incision, 3rd ICS, anterior axillary line (5-mm trocar). a, Endopath, 12-mm trocar Ethicon, USA; b, Thoracoport, $5.5 \mathrm{~mm}$-trocar Medotronic, USA. U-VATS, uniportal video-assisted thoracoscopic surgery; M-VATS, multiport video-assisted thoracoscopic surgery; ICS, intercostal space.

\section{Postoperative data}

The mean postoperative wound length was $4.2 \mathrm{~cm}$ (Figure 1). The distribution of lobectomies in both groups is shown in Figure 2. All of the pulmonary lobes were operated on in both groups (across the various patients), and most of the lobectomies in both groups were right upper lobectomies (Figure 2). The following clinical data were obtained for the U-VATS group: The median operative time was $152 \pm 19 \mathrm{~min}$, the median amount of intraoperative blood loss was $72 \pm 12 \mathrm{~mL}$, the mean number of dissected lymph nodes was $20 \pm 5$, the mean duration of the postoperative hospital stay was $7.8 \pm 1.4$ days, and the mean duration of the drainage period was $1.9 \pm 0.8$ days. There were no significant intergroup differences with regard to the operation time, intraoperative blood loss, the number of dissected lymph nodes, the duration of the drainage period, the duration of 

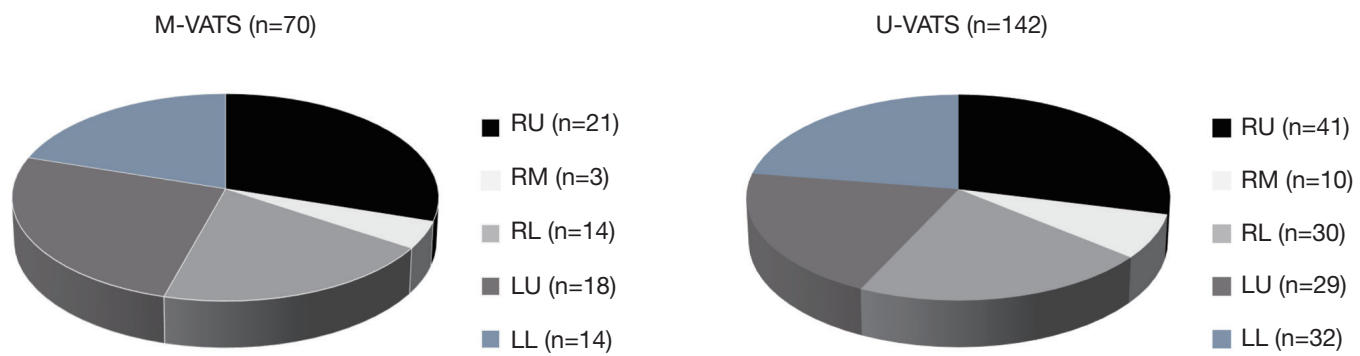

Figure 2 Distribution of lobectomy in U-VATS and M-VATS. (A) M-VATS group and (B) U-VATS group. RU, right upper; RM, right middle; RL, right lower; LU, left upper; LL, left lower; U-VATS, uniportal video-assisted thoracoscopic surgery; M-VATS, multi-port video-assisted thoracoscopic surgery.

Table 2 Postoperative outcomes

\begin{tabular}{lccc}
\hline Variables & $\begin{array}{c}\text { M-VATS } \\
(\mathrm{n}=70)\end{array}$ & $\begin{array}{c}\text { U-VATS } \\
(\mathrm{n}=142)\end{array}$ & P value \\
\hline Operation time (min) & $165 \pm 19$ & $152 \pm 18$ & 0.15 \\
Blood loss (mL) & $65 \pm 11$ & $72 \pm 12$ & 0.44 \\
The number of dissected LN & $20 \pm 5$ & $20 \pm 5$ & 0.47 \\
Drainage duration (days) & $1.8 \pm 0.9$ & $1.9 \pm 0.8$ & 0.54 \\
Hospital stay after op. (days) & $8.4 \pm 1.2$ & $7.8 \pm 1.4$ & 0.09 \\
Conversion to thoracotomy (\%) & $4(5.7)$ & $10(7.0)$ & 0.48 \\
Morbidity (\%) & $6(8.6)$ & $12(8.4)$ & 0.67 \\
Mortality (\%) & $0(0)$ & $0(0)$ & \\
\hline
\end{tabular}

Data are presented as mean \pm SD or number (percentage). Postoperative complications were including with prolonged air leak, pneumonia, atelectasis and wound infection. M-VATS, multi-port video-assisted thoracoscopic surgery; U-VATS, uniportal video-assisted thoracoscopic surgery.

the patients' hospital stays, the frequency of conversion to thoracotomy, the incidence of postoperative complications, or the mortality rate (Table 2 ).

The mean NRS score on postoperative month 1 and 2 was significantly less in the U-VATS group than in the m-VATS group (Figure 3, POM 1: $1.5 \pm 0.2$ vs. $2.9 \pm 0.3$, $\mathrm{P}<0.05$, POM 2: $0.5 \pm 0.1$ vs. $1.5 \pm 0.3, \mathrm{P}<0.01)$. During the postoperative two months, analgesics were used for a mean of $7.3 \pm 1.5$ days in the U-VATS group and $13.3 \pm 1.8$ days in the M-VATS group (Figure 4$)(\mathrm{P}<0.01)$. As for the frequency of PTPS-related symptoms on POM 2, allodynia (U-VATS: $2.1 \%$ vs. M-VATS: $10 \%, \mathrm{P}=0.028$ ), hypoesthesia ( $2.1 \%$ vs. $10 \%, \mathrm{P}=0.028)$, and numbness $(1.4 \%$ vs. $8.6 \%$,

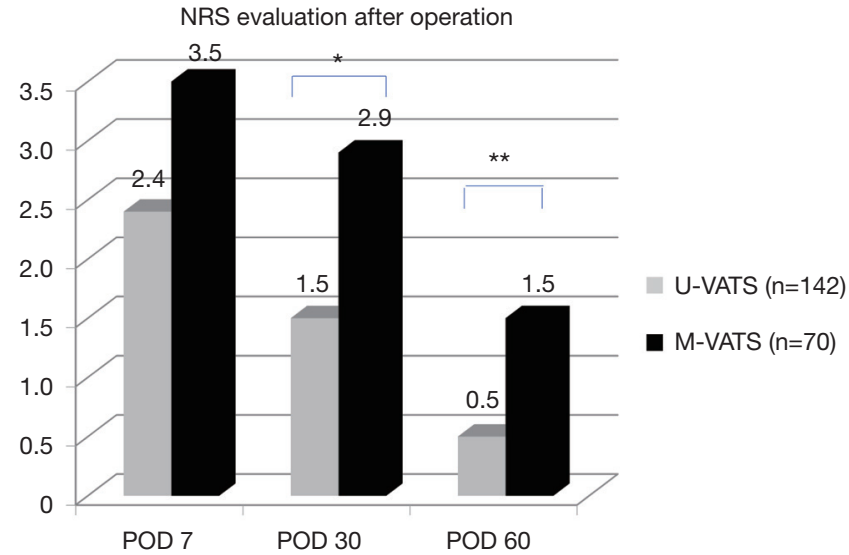

Figure 3 NRS evaluation after operation. The NRS was significantly lower in the U-VATS group than in the M-VATS group at 30 and 60 days after operation. *, $\mathrm{P}<0.05$; **, $\mathrm{P}<0.01$. NRS, numeric rating scale; POD, postoperative day; U-VATS, uniportal video-assisted thoracoscopic surgery; M-VATS, multiport video-assisted thoracoscopic surgery.

$\mathrm{P}=0.029$ ) arose in both groups (Figure 5). Aching sensation, a prickling sensation, and a burning sensation were also detected in only M-VATS groups on POM 2. Regarding the frequency of an NRS score of $>3$, the number of days on which analgesics were used, and the results of the PDQ assessment, there were no significant differences between the U-VATS and M-VATS groups. PDQ is a detective tool whether a patient has the component of neuropathic related pain. The frequency of Allodynia, hypoesthesia and numbness was significantly lower in U-VATS group than in M-VATS group. Depending on patients, there was overlapping each symptom in our study. As a result, PDQ 


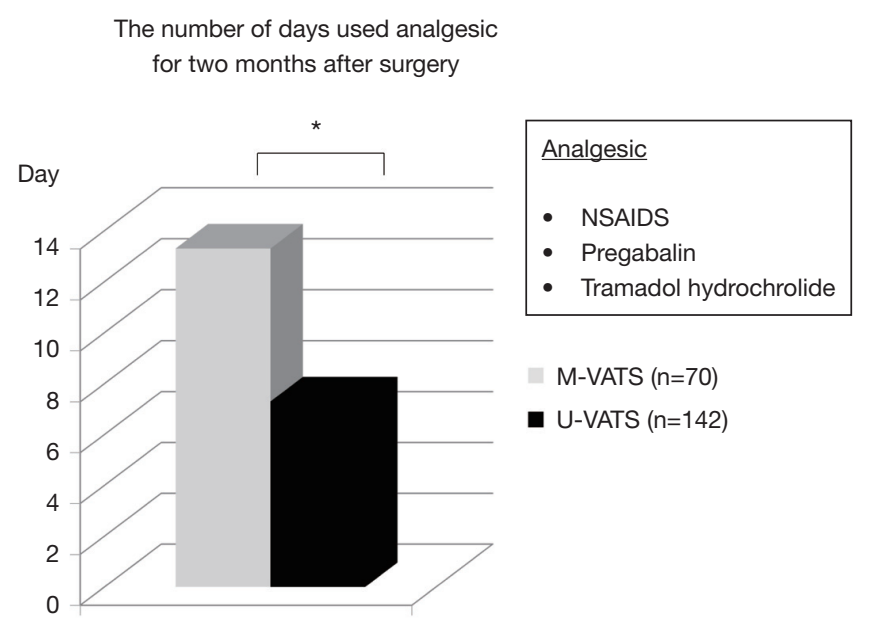

Figure 4 The number of days that were used with analgesic agents for 2 months after surgery. The number of days analgesic agents were used was significantly less in U-VATS group than in M-VATS group for 2 months after surgery. *, $\mathrm{P}<0.05$. NSAIDS, pregabalin and tramadol hydrochloride were administered as analgesic agents. U-VATS, uniportal video-assisted thoracoscopic surgery; M-VATS, multi-port video-assisted thoracoscopic surgery; NSAIDS, nonsteroid anti-inflammatory drugs.

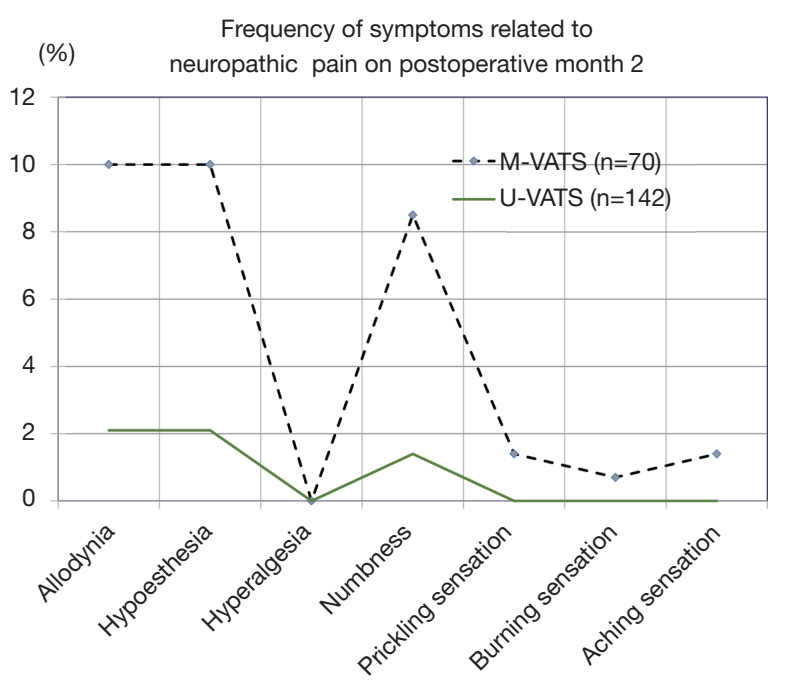

Figure 5 Frequency of symptoms related to neuropathic pain after surgery on postoperative month 2 . The occurrence of allodynia, hypoesthesia and numbness were significantly lower in U-VATS group than in M-VATS group. * $\mathrm{P}<0.05$. PDQ, pain DETECT questionnaire. U-VATS, uniportal video-assisted thoracoscopic surgery; M-VATS, multi-port video-assisted thoracoscopic surgery.
Table 3 The occurrence of PTPS in U-VATS and M-VATS

\begin{tabular}{lccc}
\hline Variables & U-VATS (N=142) & M-VATS (N=70) & P value \\
\hline NRS $>3$ & $4(2.8)$ & $5(7.1)$ & 0.268 \\
Analgesics use & $1(0.7)$ & $2(2.9)$ & 0.528 \\
PDQ (>1 symptom) & $4(2.8)$ & $7(10.0)$ & 0.059 \\
PTPS (\%) & $4(2.8)$ & $8(11.4)$ & 0.025 \\
\hline
\end{tabular}

A patient who had more than one item (NRS $>3$, Analgesic use, PDQ $>1$ symptom) on postoperative month 2 was diagnosed as PTPS. The occurrence of PTPS was significantly lower in U-VATS group than in M-VATS group. There is some overlapping in both groups. $P$ value was 0.025 . PTPS, post-thoracotomy pain syndrome; PDQ, pain DETECT questionnaire. U-VATS, uniportal video-assisted thoracoscopic surgery; M-VATS, multi-port video-assisted thoracoscopic surgery;

assessment (more than 1 symptom) showed no statistical difference between the groups. PTPS is mainly related to component of neuropathic pain, however patient's character and pathological tissue caused by the wound scarring are also influential factor of the occurrence of PTPS. We examined its pathological statement from diversified viewpoints, resulting in evaluation of three items in terms of PTPS, as shown in Table 3. As a result, the frequencies of PTPS in the U-VATS and M-VATS were $2.8 \%$ and $11.4 \%$, respectively $(\mathrm{P}=0.025)$ (Table 3).

\section{Discussion}

Although PTPS is defined as wound pain that persists for $\geq 2$ months after thoracotomy (8), there are no detailed diagnostic criteria for the condition. The mechanism responsible for PTPS is presumed to be as follows: The intercostal nerves are damaged during surgery, which induces nociceptor and inflammatory system activity and nerve cell remodeling, resulting in central sensitization. When combined with mental and social factors, this can induce PTPS (1). It is generally considered that the pain experienced after thoracic surgery includes elements of both nociceptive pain and neuropathic pain. When neuropathy persists during the wound-healing process, neuropathic pain and neuropathic pain-associated symptoms; e.g., allodynia, hypoesthesia, hyperalgesia, numbness, a prickling sensation, a burning sensation, and/or an aching sensation, can develop around the wound. In patients with PTPS, 
normally harmless (very mild) stimuli can cause abnormal sensations, such as pain (allodynia), and hypesthesia can persist in the region around the thoracotomy wound, the anterior axillary region at the intercostal thoracotomy level, and the upper abdominal region for a long time after surgery. Since at present there are no drugs that reliably alleviate the symptoms of PTPS, the associated pain, which can cause an unpleasant feeling each time clothes come into contact with the region around the wound, can reduce patients' ability to perform activities of daily living and delay their return to society. Anti-neuropathic pain agents, such as gabapentin, duloxetine, and pregabalin, are administered for PTPS; however, there is still no definitive therapy for the condition (9). The frequency of PTPS among patients that undergo thoracoscopy and thoracotomy is reported to range from approximately $18 \%$ to $50 \%$ (10). The risk factors for PTPS have been reported to include age (younger patients tend to develop more chronic pain), the presence of preoperative pain, mental distress, the presence of hyperalgesia, and handling of the intercostal nerve during invasive surgery (11). Regarding the prevention of PTPS, it is important that surgeons perform surgical procedures in a way that helps to avoid neuropathy. Since PTPS is pathologically complex, it is difficult to diagnose and evaluate it correctly. In this study, PTPS was diagnosed based on three factors related to wound pain (an NRS score of $>3$, analgesic use, and the presence of more than one of seven symptoms of neuropathic pain) and surgical factors, and the incidence of PTPS was investigated in patients with clinical stage I non-small cell lung cancer that were treated with lobectomy via U-VATS or M-VATS by the same surgeon.

No significant differences were noted in preoperative patient background or postoperative surgical factors between the U-VATS and M-VATS groups. In addition, no significant intergroup differences were detected in the NRS scores obtained early after surgery, but significant reductions in the NRS score were noted in the U-VATS group on postoperative day 30 and 60 . Though the painkillers of NSAIDS, pregabalin and tramadol that were used in our study are composite and unspecified, the total number of days on which anti-inflammatory analgesics were administered was also significantly lower in the U-VATS group during the postoperative 2 months. Regarding neuropathy-associated symptoms, three symptoms, allodynia, hypoesthesia, and numbness, were seen in both groups, but the incidence rates of all of these symptoms were significantly lower in the U-VATS group. In our
U-VATS procedure, a polyurethane wound-protecting material (the Wrap Protector mini, Hakko Inc., Japan) is applied to the wound region. Furthermore, we make a special effort to prevent damage to the intercostal nerves and muscle crushing; minimize the length of any incisions (12); and prevent intraoperative trauma to the intercostal nerves, intercostal muscles, or long thoracic nerve. In multi-port thoracoscopic surgery, 3 or 4 cylindrical trocars are placed in the intercostal region, and the intercostal nerves and/or long thoracic nerve can be accidentally crushed or damaged. In a study of the incidence rates of PTPS after thoracotomy and multi-port thoracoscopic surgery, no difference was noted between the procedures although the incidence of PTPS varied among facilities $(10,11)$. In U-VATS, both the operator and scopist use the same port, which results in a markedly smaller surgical wound and might reduce postoperative wound-derived pain.

Efforts to minimize the invasiveness of respiratory surgery have recently been progressing, and RATS and U-VATS have contributed to such efforts. However, RATS (13), which is basically a form of M-VATS, has been reported to place stress on the intercostal nerves and to be associated with high healthcare costs and a high incidence of complications. U-VATS is a thoracoscopic surgical procedure, which is performed through a $3-5-\mathrm{cm}$ thoracotomy wound. Since rib retractors are not used, and contact between the surgical instruments and the intercostal nerves is avoided as much as possible, our U-VATS procedure represents a form of minimally invasive surgery, but as U-VATS is used as a surgical treatment for lung cancer it might be necessary to investigate whether the outcomes of such treatment are acceptable. The limitation of this study is its retrospective nature and the outcomes of evaluation in terms of postoperative pain are composite. The accumulation of further experience of U-VATS is necessary to verify the postoperative incidence of PTPS, but this procedure might benefit patients.

\section{Acknowledgments}

None.

\section{Footnote}

Conflicts of Interest: The authors have no conflicts of interest to declare.

Ethical Statement: The authors are accountable for all 
aspects of the work in ensuring that questions related to the accuracy or integrity of any part of the work are appropriately investigated and resolved. The study was approved by the institutional review board and the committee of Nippon Medical School Chiba Hokusoh Hospital. Informed consent to use their data was obtained from all patients before surgery.

\section{References}

1. Kehlet H, Jensen TS, Woolf CJ. The mechanism of post-thoracotomy pain syndrome (PTPS). Lancet 2006;367:1618-25.

2. Hirai K, Takeuchi S, Usuda J. Single-incision thoracoscopic surgery and conventional video-assisted thoracoscopic surgery: a retrospective comparative study of perioperative clinical outcomes. Eur J Cardiothorac Surg 2016;49 suppl 1:i37-41.

3. Shen $Y$, Wang H, Feng $M$, et al. Single- versus multipleport thoracoscopic lobectomy for lung cancer: a propensity-matched study. Eur J Cardiothorac Surg 2016;49 Suppl 1:i48-53.

4. Wang BY, Liu CY, Hsu OK, et al. Single-incision versus multiple-incision thoracoscopic lobectomy and segmentectomy: a propensity-matched analysis. Ann Surg 2015;261:793-9.

5. Zhu Y, Liang $M, W u ~ W$, et al. Preliminary results of single-port versus triple-port complete thoracoscopic

Cite this article as: Hirai K, Usuda J. Uniportal video-assisted thoracic surgery reduced the occurrence of post-thoracotomy pain syndrome after lobectomy for lung cancer. J Thorac Dis 2019;11(9):3896-3902. doi: 10.21037/jtd.2019.09.07 lobectomy for non-small cell lung cancer. Ann Transl Med 2015;3:92.

6. Wang L, Liu D, Lu J, et al. The feasibility and advantage of uniportal video-assisted thoracoscopic surgery (VATS) in pulmonary lobectomy. BMC Cancer 2017;17:75.

7. Matsubayashi Y, Takeshita K, Sumitani M et al. Validity and reliability of the Japanese version of the painDETECT questionnaire: A multicenter observational study. PLoS One 2013:8:e68013

8. Macrae WA. Chronic post-surgical pain: 10 years on. $\mathrm{Br} \mathrm{J}$ Anaesth 2008;101:77-86.

9. Brullote V, Ruel MM, Lafontaine E, et al. Impact of pregabalin on the occurrence of postthoracotomy pain syndrome: a randomized trial. Reg Anesth Pain Med 2015;40:262-9.

10. Rogers ML, Duffy JP. Surgical aspect of chronic post-thoracotomy pain. Eur J Cardiothorac Surg 2000;18:711-16.

11. Kim W, Jesper R, Henrik K. Chronic post-thoracotomy pain: a critical review of pathogenic mechanism and strategies for prevention. Eur J Cardiothorac Surg 2009;36:170-80.

12. Hirai K, Takeuchi S, Usuda J. Single-port video-assisted thoracic surgery for early lung cancer: initial experience in Japan. J Thorac Dis 2016;8:S344-50.

13. Paul $\mathrm{S}$, Jalbert $\mathrm{j}$, Isaacs $\mathrm{AJ}$ et al. Comparative effectiveness of robotic-assisted vs thoracoscopic lobectomy. Chest 2014;146:1505-12. 